\title{
Bowel Preparation: Is Endoscopic Administration Worth the Extra Risk?
}

\author{
Yu-Hsi Hsieh • Malcolm Koo
}

Received: 12 November 2014 / Accepted: 14 November 2014/Published online: 2 December 2014

(C) Springer Science+Business Media New York 2014

Bowel preparation before colonoscopy is usually considered worse than the actual procedure. The regimen usually consists of the intake of a large amount of fluid (2-4 L) with an unpalatable taste and smell, which might lead to nausea, vomiting, bloating, and abdominal pain in addition to the required diarrhea. In a meta-analysis comparing the effectiveness of different bowel preparation agents, about $29 \%$ of the subjects were unable to ingest the full volume of polyethylene glycol (PEG) solution [1]. Incomplete consumption of the solution usually affects the quality of bowel cleansing, increasing the probability that the procedure will be aborted and then repeated.

One of the conventional ways to overcome the barrier of ingesting a large amount of an unpalatable solution is to instill it directly into the stomach with the use of a nasogastric (NG) tube. Nevertheless, shortcomings including subjects' discomfort, nasopharyngeal trauma, tube misplacement, and pulmonary aspiration have precluded its

Y.-H. Hsieh ( $\square)$

Division of Gastroenterology, Department of Medicine, Dalin

Tzu Chi Hospital, Buddhist Tzu Chi Medical Foundation, 2

Minsheng Road, Dalin, Chiayi 62247, Taiwan

e-mail: hsieh.yuhsi@msa.hinet.net

Y.-H. Hsieh

School of Medicine, Tzu Chi University, Hualien, Taiwan

M. Koo

Department of Medical Research, Dalin Tzu Chi Hospital, Buddhist Tzu Chi Medical Foundation, 2 Minsheng Road, Dalin, Chiayi 62247, Taiwan

M. Koo

Dalla Lana School of Public Health, University of Toronto,

Toronto, ON, Canada general use. It is now used only for subjects with dysphagia or with difficulty of ingesting large volume of fluids.

Delivering cathartic solutions such as PEG via an esophagogastroduodenoscope into the duodenum is a novel approach to bowel preparation. Although it is impractical to perform esophagogastroduodenoscopy (EGD) just for the sake of infusing bowel preparation regimen, same-day EGD and colonoscopy (bidirectional endoscopy) are commonly performed for the evaluation of gastrointestinal conditions, such as active gastrointestinal bleeding, iron deficiency anemia, positive fecal occult blood test, and abdominal pain. In addition to these indications, asymptomatic subjects undergo bidirectional endoscopy in the course of routine healthcare evaluation or for cancer screening. A recent US study based on a national endoscopic database showed that over $11 \%$ of subjects had received same-day bidirectional endoscopy [2]. Of the procedures, EGD is usually performed before colonoscopy due to its superior tolerability and the lower dose of sedatives needed [3]. Now, we have another reason to perform EGD before colonoscopy - to deliver the bowel preparation regimen.

In this issue of Digestive Diseases and Sciences, Jung et al. [4] evaluated the EGD-assisted administration of bowel purgative for colonoscopy. They reported that subjects who received intraduodenal PEG perfusion suffered less nausea and vomiting, felt more comfortable, and were more willing to repeat the bowel preparation method in the future than the subjects receiving oral PEG solutions. The efficacy, as judged by the Aronchick and Ottawa Scales, was comparable between the two groups. The intraduodenal method appeared safe, although more subjects who received intraduodenal $\mathrm{PEG}$ reported dizziness. Their findings are consistent with those reported in a non-randomized study by Maeng et al. [5] and in a randomized study by Barcley et al. [6]. 
The optimal timing for colonoscopy after orally administered bowel preparation is $\sim 3-4 \mathrm{~h}$ after the last dose, so that complete catharsis can be realized [7]. Theoretically, colonoscopy after EGD-administered bowel preparation can be started sooner given that the laxative has already bypassed the esophagus and the stomach. In the intraduodenal group of the current study, subjects who underwent colonoscopy within $4 \mathrm{~h}$ after EGD administration had numerically superior-defined as excellent or good-bowel preparation than those who underwent colonoscopy after $4 \mathrm{~h}$, albeit the difference was not statistically significant. Future studies with larger sample sizes should help to determine the optimal timing of colonoscopy after intraduodenal bowel preparation.

Several potential drawbacks associated with using EGD to administer bowel preparation solutions should be noted. An additional 5-15 min of procedure time is needed to infuse 500-2,000 mL of PEG, which will increase the cost and the risk of the procedure [4, 6]. For conventional bidirectional endoscopy, subjects typically complete their orally administered bowel preparation 3-4 h before the procedure and undergo colonoscopy immediately after EGD in a single sedation session. For EGD-administered bowel preparation, several hours must elapse after administration prior to colonoscopy, necessitating re-sedation and re-recovery, adding costs and risks to the procedure.

Furthermore, there are some safety concerns associated with the EGD approach. The first and most worrisome concern is the risk of aspiration. Pulmonary aspiration with life-threatening complications has previously been reported with NG administration of bowel preparation [8]. Although EGD delivers the bowel preparation solution directly to the third portion of duodenum, the purgative might still reflux back into the stomach, especially when the solution is given in a large volume within a short time. Meticulous techniques, including retracting the scope back into the stomach periodically to check and remove any refluxed fluid, should be followed during the procedure in order to minimize the risk of aspiration [6]. When a subject is sedated during EGD, the risk of aspiration is even higher. Although no aspiration-related adverse events were reported in the current study and other studies [4-6], every possible measure should be taken to minimize its risk, given its potentially catastrophic consequences.

One of the most important clinical outcomes associated with bowel preparation relates to electrolyte disturbances. The authors dissolved two pouches of PEG powder and two pouches of ascorbic acid powder in $0.5 \mathrm{~L}$ of water for intraduodenal administration. The resulting solution is hypertonic given that the contents are originally designed to be dissolved in $2 \mathrm{~L}$ of fluid. Hyperosmotic preparations can draw fluid from the intravascular space potentially causing hypovolemia and electrolyte disturbances. The increased incidence of dizziness in the intraduodenal group might be attributed to hypovolemia. The safety of intraduodenal use of such concentrated PEG solution has not been established yet.

Adequacy of hydration is another concern. Inadequate hydration appears to be an important element in the reported cases of fatal dysnatremia associated with PEG [9]. According to the pre-procedure fasting guidelines of the American Society of Anesthesiologists [10], subjects undergoing sedated EGD should fast for at least $2 \mathrm{~h}$ before and $1 \mathrm{~h}$ after the procedure, and $2 \mathrm{~h}$ before sedated colonoscopy. The prolonged fasting period coupled with fluid loss through diarrhea may deplete intravascular volume, which may be even more marked with the use of small-volume solutions such as sulfate- and magnesium-containing solutions. To minimize risk in selected subjects, intravenous hydration and electrolyte monitoring are recommended.

In conclusion, while the authors are to be commended for devising this novel bowel preparation method, the results are still far from conclusive. Although intraduodenal bowel preparation appears to reduce discomfort and achieve comparable efficacy compared with oral bowel preparation in subjects undergoing bidirectional endoscopy, it is also associated with several potential drawbacks (Table 1). More studies are required to establish the safety of the method before it can be put into widespread use. To minimize the risk of aspiration and to avoid the cost burden of repeated sedation, subjects undergoing unsedated bidirectional endoscopy can be a potential population for future studies. Furthermore, intraduodenal administration of small-volume bowel preparations can be explored since the shorter administration period is less likely to be associated with solution reflux.

Table 1 Potential indications, advantages, and disadvantages of esophagogastroduodenoscopy-assisted intraduodenal bowel preparation

\begin{tabular}{cl}
\hline $\begin{array}{c}\text { Potential } \\
\text { indications }\end{array}$ & $\begin{array}{c}\text { Bidirectional endoscopy, either on the same day or } \\
\text { on two consecutive days } \\
\text { Impaired ability to ingest large volumes of bowel } \\
\text { preparation solution }\end{array}$ \\
Advantages & $\begin{array}{c}\text { Better subject tolerance (significantly better nausea } \\
\text { and taste profiles) } \\
\text { Similar quality of bowel preparation compared with } \\
\text { oral ingestion }\end{array}$ \\
Increased risk of aspiration \\
Additional time for EGD \\
Require separate sedation sessions for EGD and \\
colonoscopy \\
Extra waiting time in hospital between EGD and \\
colonoscopy \\
Dizziness \\
Involuntary defecation during EGD
\end{tabular}

$E G D$ esophagogastroduodenoscopy 


\section{References}

1. Tan JJ, Tjandra JJ. Which is the optimal bowel preparation for colonoscopy: meta-analysis. Colorectal Dis. 2006;8:247-258.

2. Urquhart J, Eisen G, Faigel DO, Mattek N, Holub J, Lieberman DA. A closer look at same-day bidirectional endoscopy. Gastrointest Endosc. 2009;69:271-277.

3. Hsieh YH, Lin HJ, Tseng KC. Which should go first during sameday bidirectional endosocopy with propofol sedation? J Gastroenterol Hepatol. 2011;26:1559-1564.

4. Jung SW, Jung DH, Shin YC, Moh HI, Yoo H, Jang SI, Shin SR, Kim JB, Park SH, Lee MS. Transenteral bowel preparation for colonoscopy is more comfortable than the traditional method with no inferiority in efficacy. Dig Dis Sci. (Epub ahead of print). doi:10.1007/s10620-014-3344-7.

5. Maeng JH, Ko BM, Lee MS, et al. Effectiveness and tolerance of duodenoscopic bowel preparation for colonoscopy. Korean $J$ Gastroenterol. 2007;50:78-83.
6. Barclay RL. Esophagogastroduodenoscopy-assisted bowel preparation for colonoscopy. World $J$ Gastrointest Endosc. 2013;16:95-101.

7. Eun CS, Han DS, Hyun YS, et al. The timing of bowel preparation is more important than the timing of colonoscopy in determining the quality of bowel cleansing. Dig Dis Sci. 2011;56:539-544.

8. Marschall HU, Bartels F. Life-threatening complications of nasogastric administration of polyethylene glycol-electrolyte solutions (Golytely) for bowel cleansing. Gastrointest Endosc. 1998;47:408-410.

9. Ayus JC, Levine R, Arieff AI. Fatal dysnatraemia caused by elective colonoscopy. BMJ. 2003;326:382-384.

10. American Society of Anesthesiologists Task Force on Sedation and Analgesia by Non-Anesthesiologists. Practice guidelines for sedation and analgesia by non-anesthesiologists. Anesthesiology. 2002;96:1004-1017. 